\title{
Adaptive Model Predictive Control of Wave Energy Converters
}

\author{
Siyuan Zhan, Student Member, IEEE, Jing Na, Member, IEEE, Guang Li*, Member, IEEE, and Bin Wang
}

\begin{abstract}
In this paper, we propose an adaptive hierarchical model predictive control (AHMPC) scheme for wave energy converters (WECs). This AHMPC enables adaptive tuning mechanism for a model predictive control (MPC) strategy by estimating the dynamics of a WEC online, so that it can recover from performance degradation of a WEC due to the dynamics variations at different sea conditions. The proposed AHMPC consists of two layers: On the top layer, an efficient cascaded estimation algorithm is developed to online identify and update the WEC model adaptively according to the change of sea states; on the bottom layer, a specially-tailored MPC controller is implemented based on the updated WEC model to maximize the energy output subject to constraints for safe operation requirements. Numerical simulations are provided to show the efficacy of the proposed AHMPC scheme.
\end{abstract}

Index Terms-Wave energy converters, parameter estimation, model predictive control.

\section{INTRODUCTION}

Ocean waves contain enormous amount of untapped and spatially concentrated energy: $2 \mathrm{TW}$ power can be potentially extracted from ocean waves worldwide [1]. The topic on harnessing wave energy has been actively investigated over the past few decades, and many different types of wave energy converters (WECs) have been invented. Despite the great efforts, harvesting wave energy is still at an immature stage of development compared with other renewable energies, e.g. wind and solar energies, [2]-[4].

To maximize the energy output, early WEC controller design methods, e.g. latching control [5], phase control [6], declutching control [7], have been investigated based on the impedance matching principle, which suggests that a WEC controller should be designed to adaptively change the dynamics of a WEC so that its resonance frequency can match the predominant frequencies of the incoming waves [8]. Recent studies [9]-[11] show that the WEC control is essentially a constrained optimal control problem and can be tackled by model predictive control (MPC) [12], [13] or MPC-like control algorithms such as pseudospectral control [14]-[16]. Some causal optimal control strategies have also been developed

This work was supported in part by a research contract from Wave Energy Scotland's Control Systems programme, National Natural Science Foundation of China (NSFC) (No. 61573174), and a Newton Mobility Grant jointly funded by the Royal Society and NSFC (No. IE150833/ 6151101245).

Siyuan Zhan and Guang $\mathrm{Li}$ are with the School of Engineering and Materials Science, Queen Mary University of London, London, UK, E1 4NS (Email: g.li@qmul.ac.uk, s.zhan@qmul.ac.uk).

Jing $\mathrm{Na}$ and Bin Wang are with Faculty of Mechanical and Electrical Engineering, Kunming University of Science and Technology, Kunming, 650500, China. (E-mail: najing25@163.com; wangbin2173@126.com).

* Address all correspondence to Guang Li. where wave prediction is not used, so that sub-optimality can be achieved, e.g. [17], [18]. Nonlinear MPC control strategies have also been developed to tackle nonlinear effects of the WEC dynamics [14], [19]. For example, the nonlinear WEC model containing the nonlinear effects from the mooring force is online linearized for the MPC implementation in [19]; the nonlinear buoyancy force effect is explicitly incorporated into the online optimization using the flatness-based pseudospectral approach in [14]. It is known that the hydrodynamics of a WEC can vary remarkably with the change of sea conditions, especially for the frequency-dependent hydrodynamics and some nonlinear effects. Hence, as recognized in [20], the optimal operation of WECs may not be achieved with the change of sea states since the existing MPC and MPC-like WEC control algorithms are mainly designed based on a fixed dynamic model derived for a particular sea condition. To develop a high fidelity WEC model to represent the WEC dynamics in a wide range of sea conditions can be very challenging and the MPC controller based on an overly-complicated model can cause heavy computational burden for its online implementation. Especially for multi-body and multi-float WECs, the order of a control-oriented model derived from the hydrodynamic model can become very large, e.g. hundreds of states are contained in the state space model for a WEC [21]. Thus a trade-off between the modeling fidelity and complexity must be found by reducing the order of the model for controller design, which inevitably introduces the "unmodeled dynamics". The MPC controller designed without accounting for such model mismatch or uncertainties in realistic sea conditions can even drive the system's state out of its feasibility region, that is, the existing MPC or MPC-like control algorithms cannot yield a feasible solution in the presence of model mismatch. Beyond the scope of MPC schemes, there are several other control methods available to tackle the model mismatch problem. For example, a hierarchical robust control of WECs was proposed in [22] and an adaptive dynamic programming control was studied in [18] by lumping the nonlinear effects and modeling uncertainties into one term to be compensated by an estimator. More examples can be found in [23] and the references therein. However, this paper mainly focuses on the model mismatch problem in the MPC framework to directly achieve the optimal solution at various sea states.

In this paper, to address the above problem, we propose an adaptive hierarchical model predictive control (AHMPC) framework for WEC systems. On the top layer, a cascaded adaptive parameter estimation mechanism is designed to identify and update the frequency-dependent dynamics so that the WEC model can track the potential variations of the 
WEC dynamics corresponding to the change of sea states. Then the online updated dynamic model is employed by the bottom layer, where a specially-tailored MPC is designed based on the updated model to maximize the energy output and keep the constraints satisfied for safe operation purpose. In a WEC model, two frequency-dependent hydrodynamic terms corresponding to the radiation force and the wave excitation force mainly contribute to the order of the WEC model. Since in many real applications, the major source of uncertainties come from the Froude-Krylov and viscous forces contributing to the excitation force [24], we focus on the online dynamics identification for the excitation force and its associated hydrodynamics model to improve the performance of the lower level MPC controller implementation. The framework can also be extended for the online estimation of the hydrodynamics for the radiation force. Compared with the existing MPC strategies for WECs [9], [10], [12], the proposed AHMPC framework has the following advantages:

- Improved energy output performance and constraint satisfaction can be achieved when the WEC dynamics have uncertainties due to the change of sea states, compared with the MPC strategies without adaptation mechanism.

- The WEC modeling effort can be significantly reduced, which also results in a lower computational load for online controller implementation.

- A novel cascaded estimation concept is developed to explicitly estimate the dynamic parameters of wave excitation force model, which is then used to implement MPC to cope with the change of sea wave conditions.

The paper is organized as follows. Section II presents the modeling of a point absorber and its control problem. Section III introduces the cascaded adaptive estimation scheme, which enables the excitation force dynamic model to be updated online. In Section IV, we present the MPC strategy and formulate a practical implementable AHMPC framework. Simulation results are presented in Section V, and finally the paper is concluded in Section VI.

Notations: We denote the set of all real numbers by $\mathbb{R}$, the space of $n$-dimensional vectors and $m$-by- $n$ matrices by $\mathbb{R}^{n}$ and $\mathbb{R}^{m \times n}$ respectively. $\mathbb{I}_{[m, n]}$ is a set of integers from $m$ to $n$. Let $[a, b]$ denote the column vector $\left[a^{T} b^{T}\right]^{T},[A, B]$ denote matrix $\left[\begin{array}{ll}A^{T} & B^{T}\end{array}\right]^{T}, \operatorname{He}(A)$ denote $(1 / 2)\left(A+A^{T}\right), \boldsymbol{u}(t)$ and $\boldsymbol{w}(t)$ denote the $n_{p}$-steps of prediction control sequence and wave predictions at time step $t$ respectively, where $n_{p}$ is the number of wave prediction steps. The maximal and minimal eigenvalues of a square matrix $A$ are denoted by $\lambda_{\max }(A)$ and $\lambda_{\min }(A)$ respectively. $G(s)[r]$ denotes the filtered signal of $r(t)$ by a linear proper system $G(s) .\|G(s)\|_{H_{\infty}}:=$ $\sup _{\omega}|G(j w)|$ denotes the $H_{\infty}$ norm of the system $G(s)$.

\section{WEC MODELING AND PROBLEM FORMULATION}

We use a benchmark point absorber (PB) as shown in Fig. 1 as a case study, which uses the heave motion for harnessing energy. Note that the proposed AHMPC can be applied to other types of WECs without lost of generality.

For this PB, a float with a constant radius cylinder on the sea surface is linked to a piston, which can move in heave motion

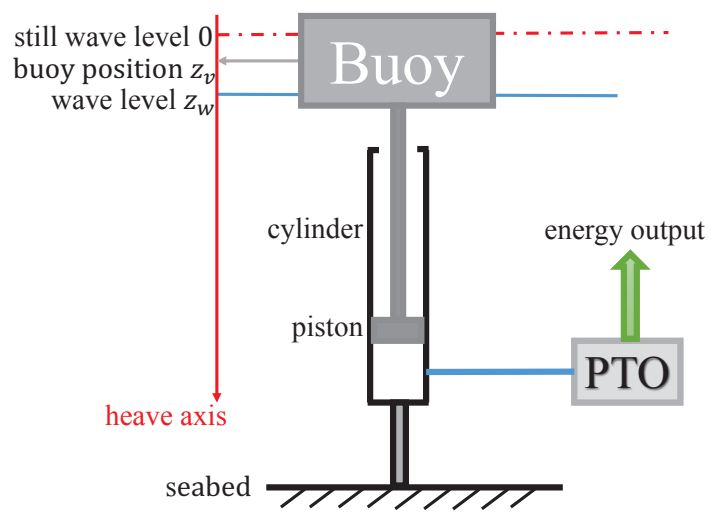

Fig. 1: Schematic diagram of the point absorber

in a cylinder fixed to the seabed. The persistent wave excitation force drives the float, which results in relative motion between the piston and the cylinder. The energy can be then captured by different power take-off (PTO) mechanisms, e.g. a direct linear generator [25], or a hydraulic motor and converter [26].

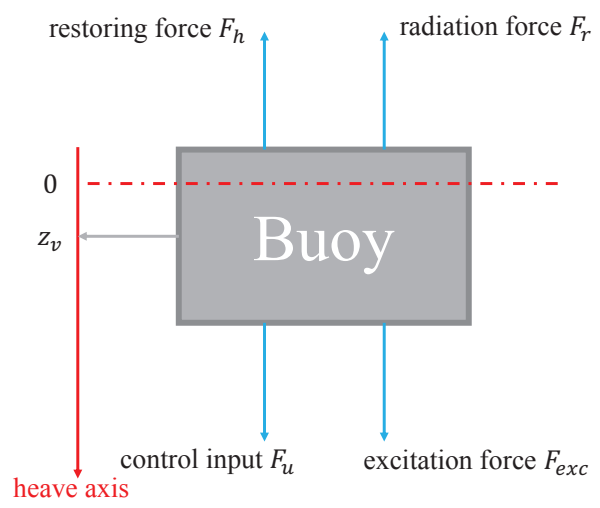

Fig. 2: Dynamics diagram of the point absorber

The free body diagram is shown in Fig. 2 and the dynamic model can be established via Newton's second law as

$$
m_{s} \ddot{z}_{v}(t)=-F_{h}(t)-F_{r}(t)+F_{e x c}(t)+F_{u}(t),
$$

where $m_{s}$ is the float mass; $z_{v}$ and $\dot{z}_{v}$ are the heave displacement and heave velocity of the float respectively; $F_{h}(t)$ is the hydrostatic restoring force calculated by

$$
F_{h}(t)=k_{s} z_{v}(t)
$$

with $k_{s}$ being the stiffness coefficient calculated by $k_{s}=\rho g S$. Here $\rho$ is the water density; $g$ is the gravitational acceleration; $S$ is the water plane area of the floating body. $F_{u}(t)$ is the PTO force acting on the piston as the control input. $F_{r}(t)$ is the radiation force, which can be represented using the Cummings equation [27] based on the linear potential theory as

$$
F_{r}(t)=F_{d}(t)+m_{\infty} \ddot{z}_{v},
$$


where $m_{\infty}$ represents the added-mass at the infinite frequency, and $F_{d}(t)$ is the convolutional term of the radiation force that can be determined by

$$
F_{d}(t)=\int_{-\infty}^{\infty} h_{r}(\tau) \dot{z}_{v}(t-\tau) d \tau,
$$

with $h_{r}(t)$ being the causal radiation impulse response. To facilitate the controller design, the convolution term (3b) can be approximated by a state-space representation as

$$
\begin{aligned}
& \dot{x}_{r}(t)=A_{r} x_{r}(t)+B_{r} \dot{z}_{v}(t) \\
& F_{d}(t) \approx C_{r} x_{r}(t)
\end{aligned}
$$

and can be parameterized using [28], [29].

Similarly, following [11], [30], the wave excitation force $F_{\text {exc }}(t)$ can be determined by

$$
F_{e x c}(t)=\int_{-\infty}^{\infty} h_{e}(\tau) z_{w}(t-\tau) d \tau
$$

with $h_{e}(t)$ as the noncausal excitation impulse response. The convolution term (4) can be approximated by a linear timevariant system $D_{e}(s)$ with a state-space realization as

$$
\begin{aligned}
\dot{x}_{e}(t) & =A_{e} x_{e}(t)+B_{e} z_{w}(t) \\
F_{e x c}(t) & \approx C_{e} x_{e}\left(t+t_{c}\right)
\end{aligned}
$$

with $t_{c}$ as the causalizing time shift [30].

With (2) and (3a), the WEC model satisfies

$$
m \ddot{z}_{v}(t)=-k_{s} z_{v}(t)-F_{d}(t)+F_{e x c}(t)+F_{u}(t),
$$

where $m:=m_{s}+m_{\infty}$ is the lumped mass.

By defining the state $x_{1}:=z_{v}, x_{2}:=\dot{z}_{v}, x:=$ $\left[x_{1}, x_{2}, x_{r}, x_{e}\right]$, disturbance input $w(t):=F_{\text {exc }}(t)$ and control input $u(t):=F_{u}(t)$, we derive a linear time variant state-space model of WEC as

$$
\dot{x}(t)=A x(t)+B_{u} u(t)+B_{w} w(t)
$$

where the matrices of the state-space model are

$$
A=\left[\begin{array}{cccc}
0 & 1 & 0 & 0 \\
-\frac{k_{s}}{m} & 0 & -\frac{C_{r}}{m} & \frac{C_{e}}{m} \\
0 & B_{r} & A_{r} & 0 \\
0 & 0 & 0 & A_{e}
\end{array}\right], B_{u}=\left[\begin{array}{c}
0 \\
\frac{1}{m} \\
0 \\
0
\end{array}\right], B_{w}=\left[\begin{array}{c}
0 \\
0 \\
0 \\
B_{e}
\end{array}\right] .
$$

With the motion dynamics as shown in Fig. 2, the extracted power at time $t$ can be expressed by

$$
P(t)=-u(t) x_{2}(t)
$$

and the extracted energy output for a period from 0 to $T$ is

$$
E(t)=\int_{0}^{T}-u(t) x_{2}(t) d t .
$$

For the safe operation of a WEC, the heave motion needs to be restricted to

$$
\left|x_{1}(t)\right| \leq \Phi_{\max },
$$

where $\Phi_{\max }$ is the heave displacement limit and the control input $u(t)$ provided by the PTO mechanism should also be constrained by

$$
|u(t)| \leq u_{\max },
$$

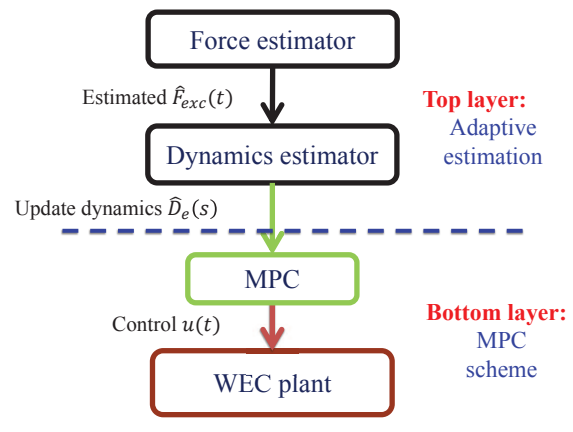

Fig. 3: Sketch of hierarchical adaptive MPC for WECs

where $u_{\max }$ is the maximal control input that can be provided by the PTO mechanism. Eqs. (11) and (12) in the WEC control problem can be treated as state and control input constraints, respectively. The satisfaction of these constraints also helps to reduce the maintenance cost and fatigue loading, and thus decreases the unit electricity cost. In summary, the WEC constrained optimal control problem can be implemented by online resolving the following optimization problem:

$$
\begin{array}{ll} 
& \min _{u(t)} \int_{0}^{T}-u(t) x_{2}(t) d t \\
\text { s.t. } & \dot{x}(t)=A x(t)+B_{u} u(t)+B_{w} w(t) \\
& \left|x_{1}(t)\right| \leq \Phi_{\max }, \quad|u(t)| \leq u_{\max } .
\end{array}
$$

Remark 1: It can be seen from (8) that the order of the WEC model is mainly contributed by the frequency-dependent dynamics. On the one hand, whilst a high fidelity model of such dynamics can cover a wide range of sea states, the large model order inevitably increases the computational burden, which may invalidate the implementation of advanced control algorithms such as MPC and MPC-like control methods. On the other hand, if the frequency dependent terms are described by a low-order model based on a particular sea state, the WEC performance degradation may occur when the sea state changes. This issue becomes even much more prominent for multi-motion and multi-float WECs (see e.g. [21]). In this paper, we assume that one of the frequency-dependent term is fixed, while the dynamics of the other term is adaptively updated so that the proposed method can cope with the dynamics variations over a large range of sea states.

\section{CASCADED ESTIMATION FOR EXCITATION DYNAMICS}

In this section we propose a cascaded estimation method to estimate the dynamic model associated with the excitation force for demonstration purpose, since the modeling uncertainties may mainly come from the Froude-Krylov and viscous forces [24] . Note that the method can also be extended to the estimation of the dynamic model associated with the radiation force. The technical challenge lies in the fact that the excitation force $F_{\text {exc }}(t)$ is not directly measurable. Hence, we first design a dynamic estimator to estimate $F_{\text {exc }}(t)$ by using $x_{1}, x_{2}, u, x_{r}$, which is followed by another adaptive parameter estimator to approximate the convolutional term corresponding to the 
frequency-dependent excitation force dynamics $D_{e}(s)$, i.e. $A_{e}, B_{e}, C_{e}$. In this case, we assume the dynamics associated with the radiation force is not changing significantly and thus radiation force can be calculated and used for the estimation of the excitation force $F_{\text {exc }}(t)$. In the simulation, we will show how this inaccurate estimation of the radiation force can influence the control performance.

\section{A. Estimation of excitation force $F_{\text {exc }}(t)$}

To estimate the convolutional term of the excitation force $F_{\text {exc }}(t)$, we find from (7) that the unknown excitation force $F_{\text {exc }}(t)$ can be taken as a 'virtual input' of the system. Hence, inspired by [31], we can further tailor the principle of unknown input observer and propose an adaptive estimator framework for $F_{\text {exc }}(t)$, whose framework is shown in Fig. 4.

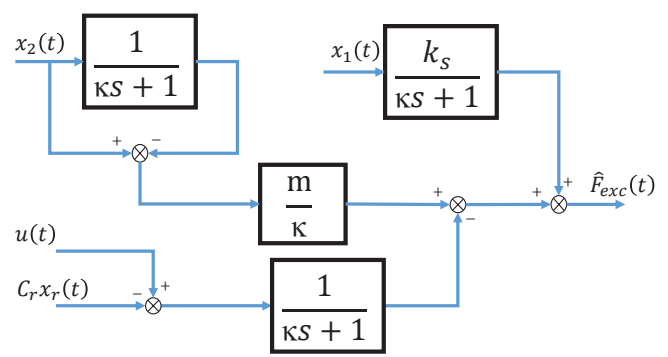

Fig. 4: Adaptive estimator framework for $\hat{F}_{\text {exc }}(t)$ in (14)

Lemma 1: The estimated excitation force $\hat{F}_{\text {exc }}(t)$ constructed by

$$
\hat{F}_{\text {exc }}:=\frac{m}{\kappa}\left(x_{2}-\frac{1}{\kappa s+1}\left[x_{2}\right]\right)+\frac{k_{s}}{\kappa s+1}\left[x_{1}\right]-\frac{1}{\kappa s+1}[\mu]
$$

can exponentially converge to its true value $F_{\text {exc }}(t)$ provided that the filter constant $\kappa>0$ is designed to be small enough. Here $\mu$ denotes the lumped variable $\mu:=u-C_{r} x_{r}$.

Proof: With $\mu:=u-F_{r}$, Eq. (7) can be rewritten as

$$
m \dot{x}_{2}=-k_{s} x_{1}+F_{e x c}+\mu .
$$

By applying a low-pass filter $1 /(\kappa s+1)$ on both sides of (15), we have

$$
m \dot{x}_{2 g}=-k_{s} x_{1 g}+F_{e x c, g}+\mu_{g},
$$

where $x_{2 g}:=\frac{1}{\kappa s+1}\left[x_{2}\right], x_{1 g}:=\frac{1}{\kappa s+1}\left[x_{1}\right], \mu_{g}:=\frac{1}{\kappa s+1}[\mu]$ and $F_{e x c, g}:=\frac{1}{\kappa s+1}\left[F_{e x c}\right]$, respectively. From the notation for the filter, we can verify that $\dot{x}_{2 g}:=\frac{x_{2}-x_{2 g}}{\kappa}$. By comparing (16), (15) and (14), we can verify that the proposed estimator is equivalent to the filter version of $F_{\text {exc }}(t)$, that is

$$
\hat{F}_{\text {exc }}(t)=F_{\text {exc }, g} \text {. }
$$

From the property of the filter $1 /(\kappa s+1)$, we can represent the estimator given by $F_{e x c, g}:=\frac{1}{\kappa s+1}\left[F_{e x c}\right]$ in the time-domain as

$$
\dot{F}_{e x c, g}=-\frac{1}{\kappa} F_{e x c, g}+\frac{1}{\kappa} F_{e x c},
$$

which gives the estimation error $e_{F_{e x c}}:=F_{e x c}-\hat{F}_{e x c, g}$ as

$$
\dot{e}_{F_{e x c}}=\dot{F}_{e x c}-\dot{F}_{e x c, g}=-\frac{1}{\kappa} e_{e x c}+\dot{F}_{d} .
$$

Then by choosing a Lyapunov function as $V=\frac{1}{2} e_{e x c}^{2}$, the derivative of $V$ can be computed by

$\dot{V}=e_{F_{e x c}} \dot{e}_{F_{e x c}} \leq-\frac{1}{\kappa} e_{F_{e x c}}^{2}+\left|e_{F_{e x c}}\right| \varpi \leq-\frac{1}{2 \kappa} e_{F_{e x c}}^{2}+\frac{\kappa}{2} \varpi_{(20)}^{2}$,

where $\varpi:=\sup \left|\dot{F}_{\text {exc }}(t)\right|$ defines the upper bound of the radiation force variation. The above inequality implies that $V(t) \leq e^{-\frac{t}{\kappa}} V(0)+\frac{\kappa^{2}}{2} \varpi^{2}$. Consequently, we can obtain from the definition of $V$ that

$$
\left|e_{F_{e x c}}(t)\right| \leq \sqrt{e^{-\frac{t}{\kappa}} e_{F_{e x c}}^{2}(0)+\kappa^{2} \varpi^{2}}
$$

which further indicates that $e_{F_{e x c}} \rightarrow 0$ exponentially as $\kappa \rightarrow 0$. This completes the proof.

Lemma 1 shows that the excitation force $F_{\text {exc }}(t)$ can be online estimated via the proposed estimator (14), where fast (exponential) convergence can be strictly achieved. This tackles the difficulty in identifying the excitation force in (6). Hence, the estimated force $\hat{F}_{\text {exc }}$ can be used in the identification of the excitation force dynamics $D_{e}(s)$ in the next subsection.

\section{B. Adaptive dynamics estimation (ADE) of excitation force}

From the excitation force given in (6) and the analysis in [30], the excitation force dynamics $D_{e}(s)$ can be represented by

$$
D_{e}(s)=\frac{\hat{F}_{e x c}}{w}=\frac{b_{m} s^{m}+\cdots+b_{1} s+b_{0}}{s^{n+1}+a_{n} s^{n}+\cdots+a_{1} s+a_{0}} .
$$

Hence, the problem to be addressed is to estimate the unknown coefficients $a_{i}, b_{j}$ for $i=0,1, \ldots, n$ and $j=$ $0,1, \ldots, m, n \geq m$ in the above excitation force dynamics. To implement the online estimation algorithm, we present the above dynamics in the time-domain. For this purpose, we select a Hurwitz polynomial $\Lambda(s)=s^{n+1}+\lambda_{n} s^{n}+\cdots+\lambda_{1} s+\lambda_{0}$, and then filter $(21)$ by $1 / \Lambda(s)$ as

$$
\begin{aligned}
& \frac{s^{n+1}}{\Lambda(s)}\left[\hat{F}_{e x c}\right]+a_{n} \frac{s^{n}}{\Lambda(s)}\left[\hat{F}_{e x c}\right]+\cdots+a_{0} \frac{1}{\Lambda(s)}\left[\hat{F}_{e x c}\right] \\
& \quad=b_{m} \frac{s^{m}}{\Lambda(s)}[w]+\cdots+b_{0} \frac{1}{\Lambda(s)}[w] .
\end{aligned}
$$

Adding $-\frac{\Lambda(s)}{\Lambda(s)}\left[\hat{F}_{\text {exc }}\right]$ to both sides of (22), we can obtain the parameterized form as

$$
\hat{F}_{e x c}=\Theta^{T} \Phi_{f}
$$

where

$$
\begin{aligned}
\Theta:= & {\left[\lambda_{n}-a_{n}, \ldots, \lambda_{1}-a_{1}, \lambda_{0}-a_{0}, b_{m}, \ldots, b_{1}, b_{0}\right]^{T} } \\
\Phi_{f}:= & {\left[\frac{s^{n}}{\Lambda(s)}\left[\hat{F}_{\text {exc }}\right], \ldots, \frac{s}{\Lambda(s)}\left[\hat{F}_{\text {exc }}\right], \frac{1}{\Lambda(s)}\left[\hat{F}_{\text {exc }}\right],\right.} \\
& \left.\frac{s^{m}}{\Lambda(s)}[w], \ldots, \frac{s}{\Lambda(s)}[w], \frac{1}{\Lambda(s)}[w]\right]^{T} .
\end{aligned}
$$

Eq. (23) shows that the unknown parameter vector $\Theta$ is in a linearly parameterized form with $\hat{F}_{\text {exc }}(t)$ and $w(t)$ as the output and input respectively. Then we can develop the 


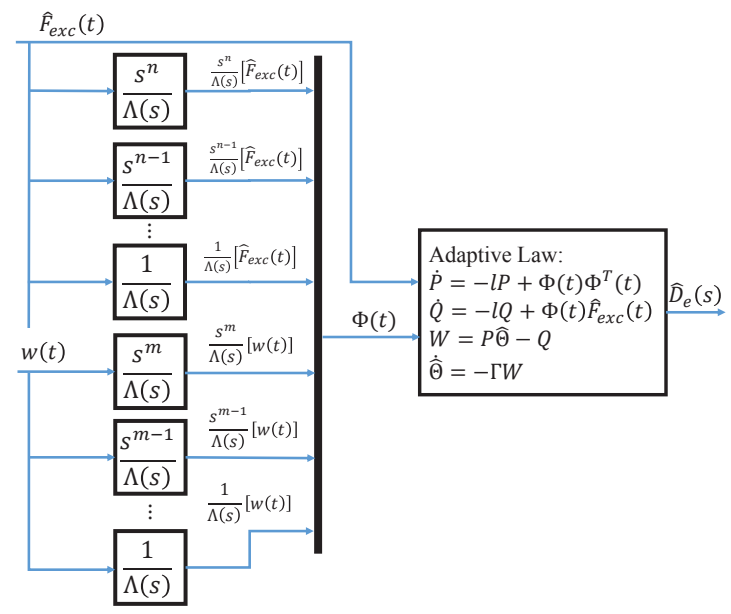

Fig. 5: Adaptive estimator framework for excitation dynamic $D_{e}(s)$

following adaptive parameter estimation scheme to online estimate $\Theta$.

Denote the auxiliary matrix $P$ and auxiliary vector $Q$ as follows

$$
\left\{\begin{aligned}
\dot{P} & =-l P+\Phi_{f} \Phi_{f}^{T}, & P(0)=0 \\
\dot{Q} & =-l Q+\Phi_{f} \hat{F}_{e x c}, & Q(0)=0
\end{aligned}\right.
$$

where $l>0$ is a forgetting factor parameter to be designed.

The adaptive law for updating the unknown parameter $\hat{\Theta}$ is proposed as

$$
\dot{\hat{\Theta}}=-\Gamma W,
$$

where $W:=P \hat{\Theta}-Q$ and $\Gamma>0$ is the learning gain.

To prove the convergence of the adaptive algorithm (25), the following lemma is needed:

Lemma 2: The variable $W:=P \hat{\Theta}-Q$ used in (25) can be equivalently represented as

$$
W=-P \tilde{\Theta},
$$

where $\tilde{\Theta}=\Theta-\hat{\Theta}$ is the estimation error.

Proof: One can solve the matrix equation (24) and obtain its solution as

$$
\left\{\begin{array}{l}
P(t)=\int_{0}^{t} e^{-l(t-r)} \Phi_{f}(r) \Phi_{f}^{T}(r) d r \\
Q(t)=\int_{0}^{t} e^{-l(t-r)} \Phi_{f}(r) \hat{F}_{d}(r) d r
\end{array}\right.
$$

From (27), we may find that $Q=P \Theta$. Then by substituting $Q=P \Theta$ into $W=P \hat{\Theta}-Q$, one can obtain (26).

It is clearly shown in Lemma 2 that the derived variable $W$ in the adaptive law includes the parameter estimation error $\tilde{\Theta}$, such that the estimation convergence can be achieved using the adaptive law (25) driven by the variable $W$.

Before proving the convergence of the proposed adaptive law, we need to investigate the positive definiteness of the matrix $P$.

Lemma 3: [32] The condition $\lambda_{\min }(P)>\sigma>0$ holds for any constant $\sigma>0$ (i.e. $P$ is positive definite) provided that the regressor $\Phi_{f}$ is persistently excited (PE).

The main convergence property of the proposed adaptive law (25) can be given as follows:
Theorem 1: Consider the adaptive law (25) and the regressor $\Phi_{f}$ is PE, the estimation error $\tilde{\Theta}$ exponentially converges to zero.

Proof: We select a Lyapunov function as $V=$ $\frac{1}{2} \tilde{\Theta}^{T} \Gamma^{-1} \tilde{\Theta}$, and calculate its time derivative along (25) as

$$
\dot{V}=\tilde{\Theta}^{T} \Gamma^{-1} \dot{\tilde{\Theta}}=-\tilde{\Theta}^{T} P \tilde{\Theta} \leq-\sigma\|\tilde{\Theta}\|^{2} \leq-\mu V,
$$

where $\mu=2 \sigma / \lambda_{\max }\left(\Gamma^{-1}\right)$ denotes a positive constant. Now, from the Lyapunov's Theorem, we know that $\tilde{\Theta}$ can exponentially converge to zero. Consequently, one can find that the estimated parameter $\hat{\Theta}$ converges to their true value $\Theta$.

Using the estimated parameter $\hat{\Theta}$, one can calculate the coefficients $a_{i}, b_{j}$ embedded in the excitation force dynamics based on (23). Hence, the online estimation of the unknown excitation dynamics has been achieved.

\section{MPC DESIGN AND PRACTICAL CONSIDERATIONS}

\section{A. MPC formulation}

In this section, we design a MPC controller at the bottom layer of the proposed AHMPC framework to maximize the energy output while handling the state and input constraints. Using the estimated radiation force dynamics, we have the online updated WEC model

$$
\dot{x}(t)=\hat{A}(t) x(t)+B_{u} u(t)+\hat{B}_{w}(t) w(t),
$$

where

$$
\hat{A}=\left[\begin{array}{cccc}
0 & 1 & 0 & 0 \\
-\frac{k_{s}}{m} & 0 & -\frac{C_{r}}{m} & \frac{\hat{C}_{e}}{m} \\
0 & B_{r} & A_{r} & 0 \\
0 & 0 & 0 & \hat{A}_{e}
\end{array}\right], B_{u}=\left[\begin{array}{c}
0 \\
\frac{1}{m} \\
0 \\
0
\end{array}\right], \hat{B}_{w}=\left[\begin{array}{c}
0 \\
0 \\
0 \\
\hat{B}_{e}
\end{array}\right]
$$

with $\hat{A}_{e}, \hat{B}_{e}$ and $\hat{C}_{e}$ as the corresponding state-space representation of the estimated excitation dynamics $\hat{D}_{e}(s)$.

To implement the MPC controller, the updated WEC model needs to be discretized with sampling time $t_{s}$ into

$$
x(k+1)=A_{d} x(k)+B_{u d} u(k)+B_{w d} w(k) .
$$

To achieve non-casual control, $t_{p}$ seconds of wave elevation prediction are assumed to be available, which can be obtained using a short term wave forecasting technique, e.g. autoregressive (AR) [33]. Here, $n_{p}$ satisfies $t_{p}=n_{p} t_{s}$ for sampling interval $t_{s}$. The MPC strategy in the bottom layer can be reformulated as

$$
\begin{array}{ll} 
& \min _{\boldsymbol{u}} \sum_{k=0}^{n_{p}-1} v(k) u(k)+q z^{2}(k)+r u^{2}(k) \\
\text { s.t. } & x(k+1)=A_{d} x(k)+B_{u d} u(k)+B_{w d} w(k) \\
& z(k)=C_{z} x(k), v(k)=C_{v} x(k) \\
& |z(k)| \leq \Phi_{\max }, \quad|u(k)| \leq u_{\max }, \quad \text { for } k \in \mathbb{I}_{\left[0, n_{p}-1\right]},
\end{array}
$$

where $C_{z}:=\left[\begin{array}{lll}1 & 0 & 0_{1 \times\left(n_{r}+n_{e}\right)}\end{array}\right] ; C_{v}:=\left[\begin{array}{lll}0 & 1 & 0_{1 \times\left(n_{r}+n_{e}\right)}\end{array}\right] ;$ $\Phi_{\max }$ and $u_{\max }$ are the heave displacement limit and control input limit defined in (11) and (12), respectively. Note that similar to [11], [12], a modified stage cost is adopted, where the first term $-v(k) u(k)$ represents the power at time $k$ that 
can be absorbed by the PTO mechanism; the last two terms $q z^{2}(k)+r u^{2}(k)$ are used to penalize the heave displacement $z(k)$ and control input $u(k)$ respectively; the weights $q$ and $r$ are tuning parameters to guarantee the convexity of cost (31a) and also influence the feasibility and stability of the system. More details on choosing the weights $q$ and $r$ can be found in [11], [12].

To implement the MPC, the optimization problem (31) is converted into a quadratic programming $(\mathrm{QP})$ problem in the following. The predicted state trajectory at time $t$ can be expressed by

$$
x(t+k \mid t)=A_{d}^{k} x(t)+\mathcal{M}_{k} \boldsymbol{u}(t)+\mathcal{C}_{k} \boldsymbol{w}(t)
$$

for $k \in \mathbb{I}_{\left[0, n_{p}-1\right]}$, where $\mathcal{M}_{k} \in \mathbb{R}^{n_{x} \times n_{p}}$ and $\mathcal{C}_{k} \in \mathbb{R}^{n_{x} \times n_{p}}$ are defined by

$$
\begin{aligned}
\mathcal{M}_{k} & :=\left[\begin{array}{lllllll}
A_{d}^{k-1} B_{u d} & A_{d}^{k-2} B_{u d} & \ldots & B_{u d} & 0 & \ldots & 0
\end{array}\right] \\
\mathcal{C}_{k} & :=\left[\begin{array}{lllllll}
A_{d}^{k-1} B_{w d} & A_{d}^{k-2} B_{w d} & \ldots & B_{w d} & 0 & \ldots & 0
\end{array}\right] .
\end{aligned}
$$

Lemma 4: The optimization problem (31) can be solved by the following QP

$$
\begin{gathered}
\boldsymbol{u}^{*}(t)=\underset{\boldsymbol{u}(t)}{\arg \min } \boldsymbol{u}^{T} \mathcal{H} \boldsymbol{u}(t)+\boldsymbol{u}(t)^{T} \mathcal{F} \\
\text { s.t. } \mathcal{A} \boldsymbol{u}(t) \leq \mathcal{B} .
\end{gathered}
$$

where the coefficients $\mathcal{H} \in \mathbb{R}^{n_{p} \times n_{p}}, \mathcal{F} \in \mathbb{R}^{n_{p} \times 1}, \mathcal{A} \in \mathbb{R}^{4 n_{p} \times 1}$ and $\mathcal{B} \in \mathbb{R}^{4 n_{p} \times 1}$ are defined by

$$
\begin{aligned}
\mathcal{H}:= & r I_{n_{p}}+\operatorname{He}\left(\left[C_{v} \mathcal{M}_{0}, C_{v} \mathcal{M}_{1}, \ldots, C_{v} \mathcal{M}_{n_{p}-1}\right]\right) \\
& +q \sum_{k=0}^{n_{p}-1}\left(C_{z} \mathcal{M}_{k}\right)^{T} C_{z} \mathcal{M}_{k} \\
\mathcal{F}:= & q \sum_{k=0}^{n_{p}-1}\left(C_{z} \mathcal{M}_{k}\right)^{T} \mathcal{L}_{k}+\left[\mathcal{K}_{0}, \mathcal{K}_{1}, \ldots, \mathcal{K}_{n_{p}-1}\right] \\
\mathcal{A}:= & {\left[\begin{array}{c}
C_{z} \mathcal{M}_{0} \\
\vdots \\
C_{z} \mathcal{M}_{n_{p}-1} \\
-C_{z} \mathcal{M}_{0} \\
\vdots \\
-C_{z} \mathcal{M}_{n_{p}-1} \\
I_{n_{p}} \\
-I_{n_{p}}
\end{array}\right], \mathcal{B}:=\left[\begin{array}{c}
\Phi_{\max }-\mathcal{L}_{0} \\
\vdots \\
\Phi_{\max }-\mathcal{L}_{n_{p}-1} \\
\Phi_{\max }+\mathcal{L}_{0} \\
\vdots \\
\Phi_{\max }+\mathcal{L}_{n_{p}-1} \\
u_{\max } 1_{2 n_{p} \times 1}
\end{array}\right] }
\end{aligned}
$$

where $\mathcal{K}_{k}:=C_{v} A_{d}^{k} x(t)+C_{v} \mathcal{C}_{k} \boldsymbol{w}(t) ; \mathcal{L}_{k}:=C_{z} A_{d}^{k} x(t)+$ $C_{z} \mathcal{C}_{k} \boldsymbol{w}(t) ; 1_{n_{p} \times 1}$ is a $n_{p}$-by-1 column vector with each element as $1 ; \mathcal{M}_{k}$ and $\mathcal{C}_{k}$ are defined in (33); $C_{v}$ and $C_{z}$ are defined in (31).

Proof: The proof can be shown by some straightforward matrix manipulations. See e.g. [11], [12] for more details.

\section{B. Observer design and implementation of AHMPC}

In Subsection IV-A, the MPC design is based on the assumption that full information of state $x(t)$ is available at time $t$. However, the states associated with the radiation dynamics $D_{r}(s)$ and excitation dynamics $D_{e}(s)$ are not directly measurable and in this case only the heave displacement and

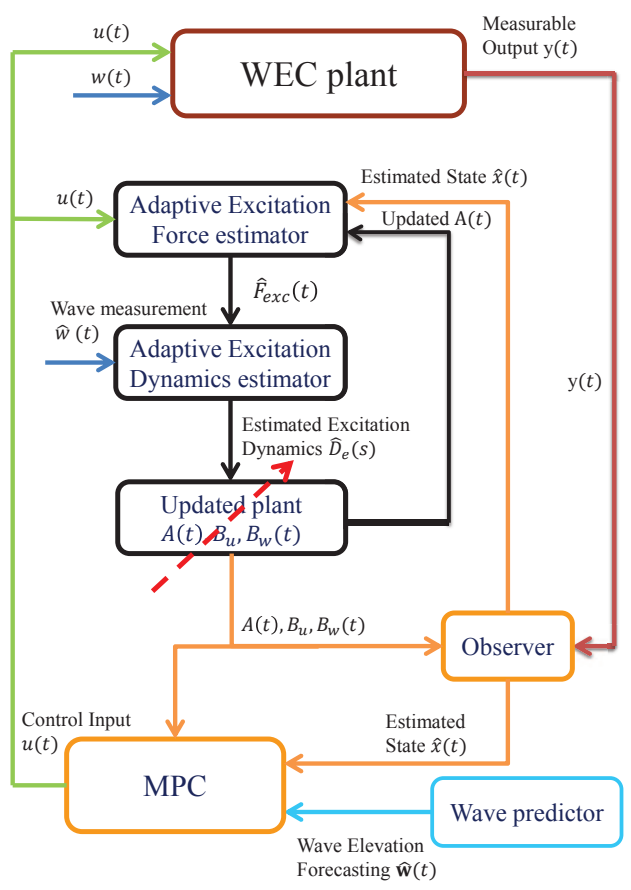

Fig. 6: the proposed adaptive MPC framework

heave velocity are assumed to be measurable, i.e. the measured output is

$$
y(t)=C x(t)
$$

where $C:=\left[I_{2}, 0_{2 \times\left(n_{r}+n_{e}\right)}\right]$. The pair $(A, C)$ are assumed to be observable. To estimate the full state information, we design a Luenberger observer

$$
\dot{\hat{x}}(t)=\hat{A} \hat{x}(t)+B_{u} u(t)+B_{w} w(t)+L(y(t)-C x(t)) .
$$

Here $\hat{x}(t)$ is the estimated state and $L$ is the observer gain to be properly designed such that after some "warm-up period", the difference between the actual state $x(t)$ and estimated state $\hat{x}(t)$ becomes negligible. The proof of the convergence of the above observer can be found in the existing literatures, e.g. [11], [12]. The implementation of the proposed AHMPC framework is schematically illustrated in Fig. 6.

\section{Simulation}

In this section, we present numerical simulations to show the efficacy of the proposed AHMPC framework. The model parameters of the PB to be studied are the same as those in [30] and are summarized in Table I.

TABLE I: The parameters used for the PB model

\begin{tabular}{lll}
\hline Description & Notation & values \\
\hline Stiffness & $k_{s}$ & $3866 \mathrm{~N} / \mathrm{m}$ \\
Float mass & $m_{s}$ & $242 \mathrm{~kg}$ \\
Added mass & $m_{a}$ & $83.5 \mathrm{~kg}$ \\
Total mass & $m$ & $325.5 \mathrm{~kg}$ \\
Input force limit & $u_{\max }$ & $1500 \mathrm{~N}$ \\
Heave displacement limit & $\Phi_{\max }$ & $1 \mathrm{~m}$ \\
\hline
\end{tabular}


The dynamics of radiation force can be expressed by

$$
D_{r}(s)=\frac{75.1 s^{2}+394 s+36.5}{1+4.41 s^{2}+17.7 s+17.9} .
$$

To demonstrate the robustness of the proposed AHMPC framework, we deliberately introduce model mismatch caused by the variation of dynamics for modeling excitation force with the change of sea states in the simulations. We assume the accurate transfer function of the impulse function of excitation force is

$$
D_{e}(s)=\frac{313 s^{2}-3425 s+23945}{1+3.24 s^{2}+7.22 s+5.57}
$$

and the inaccurately modeled excitation force dynamics are assumed to be

$$
\bar{D}_{e}(s)=\frac{280 s^{2}-3025 s+21515}{1+4.91 s^{2}+9.03 s+5.6} .
$$

Here we assume the modeling uncertainties due to the model order reduction and the nonlinear effects are lumped together and these uncertainties become prominent for a typical range of sea wave periods. The parameters are deliberately chosen in (41) to reflect such a modeling mismatch.

We next show the necessity for using the proposed cascaded estimator to address the modeling uncertainties and their influence on the control. For this purpose, simulations with three different controllers are compared:

1) With model mismatch, MPC with adaptive estimation of excitation force dynamics, which is referred as "with ADE".

2) With model mismatch, MPC without adaptive estimation of excitation force dynamics, where only the nominal model (41) with modeling uncertainties is used in the control, which is referred as "without ADE".

3) No model mismatch, MPC with the accurate model of excitation force dynamics $D_{e}(s)$ (40), which is referred as "no model mismatch".

A 500 second sea wave generated with JONSWAP spectrum is used [34], whose corresponding wave elevation profile is shown in Fig. 7. The peak wave period and significant wave height are 3 second and $1 \mathrm{~m}$, respectively.

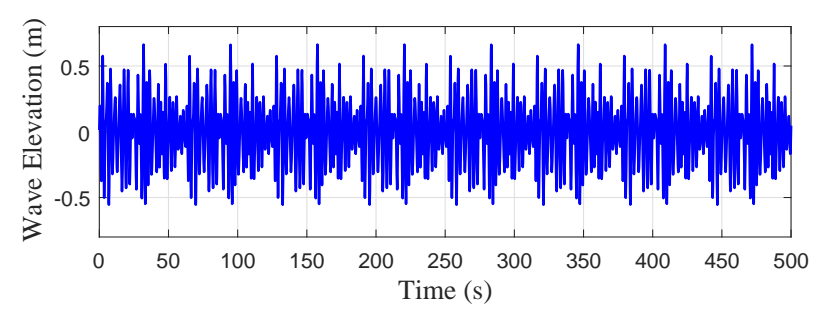

Fig. 7: Wave elevation profile

The low-pass filter of the excitation force estimator (14) is implemented with $\kappa=0.015$. The polynomial used in the adaptive excitation force dynamics estimation (22) is selected as $\Lambda(s)=s^{3}+30 s^{2}+300 s+1000$, and the parameters in the proposed adaptive law for excitation force dynamics estimation are selected as: $l=0.05 ; \Gamma=$

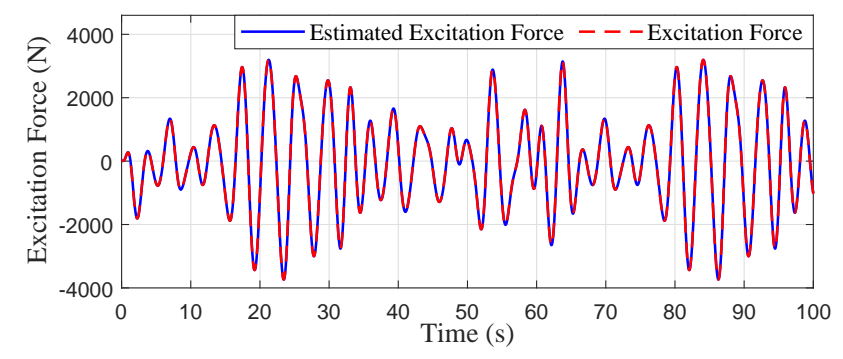

Fig. 8: Excitation force estimation

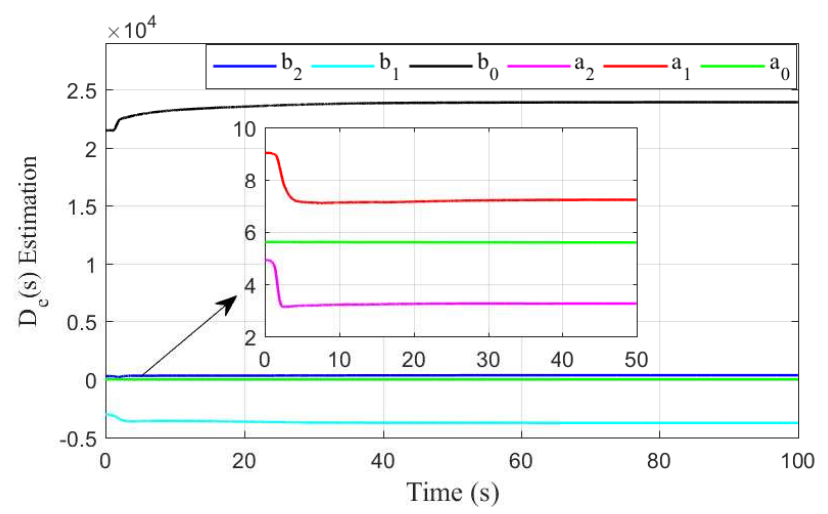

Fig. 9: Adaptive excitation dynamics $D_{e}(s)$ Estimation

$\operatorname{diag}(0.4,0.6,0.04,20000,100000,800000)$. The parameters used in the MPC are set as $q=100, r=1 \times 10^{-4}$.

Fig. 8 shows the profiles of the excitation force estimation, which indicates that the proposed excitation force estimation is accurate. Based on the estimated excitation force and the measured heave velocity $x_{2}$, an adaptive estimator is applied to estimate the excitation force dynamics. Fig 9 shows the excitation force dynamics estimation results, which indicate that the unknown coefficients in $D_{e}(s)$ can be precisely estimated online after a transient convergence period around $30 \mathrm{~s}$.

Fig. 10 shows the $H_{\infty}$ norm difference between the true excitation force dynamics and the estimated excitation force dynamics, $\left\|D_{e}(s)-\hat{D}_{e}(s)\right\|_{H_{\infty}}$, which is used to measure the model accuracy of excitation force dynamics (this method was used in [35] to measure the accuracy of hydrodynamic model). We can see that $\left\|D_{e}(s)-\hat{D}_{e}(s)\right\|_{H_{\infty}}$ is reduced from 1401

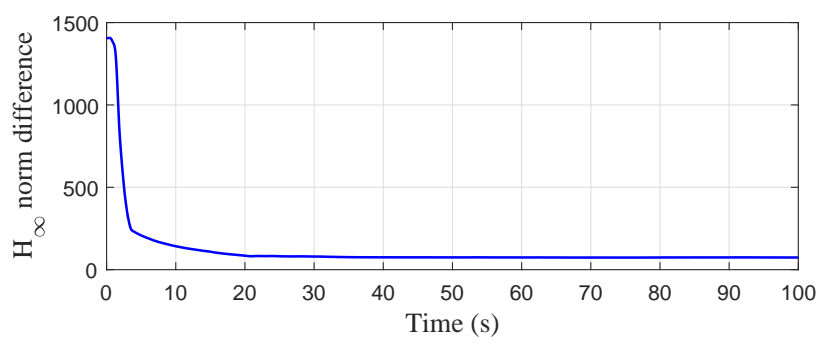

Fig. 10: $H_{\infty}$ norm difference between the true excitation force dynamics $D_{e}(s)$ and the estimated excitation force dynamics $\hat{D}_{e}(s) .\left(\left\|D_{e}(s)-\hat{D}_{e}(s)\right\|_{H_{\infty}}\right)$. [35] 
to 82 in the first $30 \mathrm{~s}$, which represents a decrease of $94.15 \%$ modeling error. Fig. 11 shows the bode diagram of the accurate $D_{e}(s)$ described in (40), the inaccurate $\bar{D}_{e}(s)$ described in (41) (Blue) and $\hat{D}_{e}(s)$ corrected using the proposed ADE mechanism for $30 \mathrm{~s}$ (Red). We can see the model mismatch is significantly reduced, especially between 1-10 rad/s. Figs. 1011 indicates that the proposed cascaded estimation approach can reconstruct the dynamics of unmeasurable excitation force rapidly, which in turn can help to improve the control performance when it is incorporated into the MPC design.

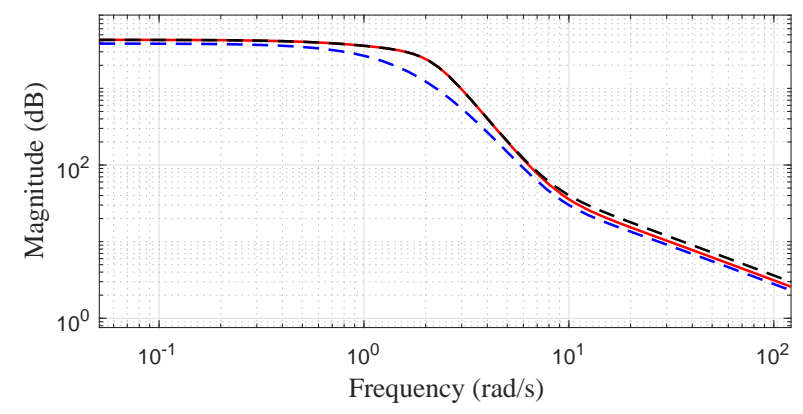

Fig. 11: Bode diagram of accurate $D_{e}(s)$ (40) (Red); inaccurate $\bar{D}_{e}(s)$ (41) (Blue); corrected $\hat{D}_{e}(s)$ after using ADE mechanism for $30 \mathrm{~s}$ (Black).

Fig. 12 shows the states and control input responses with the proposed MPC scheme. We can see that both input and state constraints are strictly satisfied with the proposed adaptive MPC method. Finally, Fig. 13 provides the extracted energy output of the above mentioned three control methods respectively. One can find that with the help of the proposed adaptation element, the proposed AHMPC with estimators (Case 1) can achieve almost the same energy output as that with precise WEC model information (Case 3), whilst the MPC with only a fixed nominal model (Case 2) leads to significantly reduced energy output. All of these simulation results verify the efficacy of the proposed AHMPC scheme.
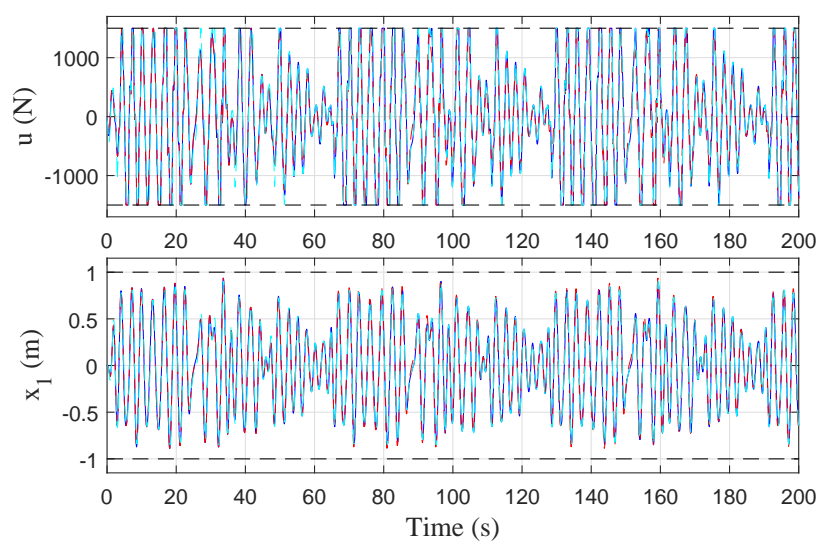

Fig. 12: State and Input response: "With ADE" (Blue); "Without ADE" (Red); "No model mismatch" (Cyan).

To further test the robustness of the AHMPC subject to the radiation force dynamics uncertainties, we intentionally intro-

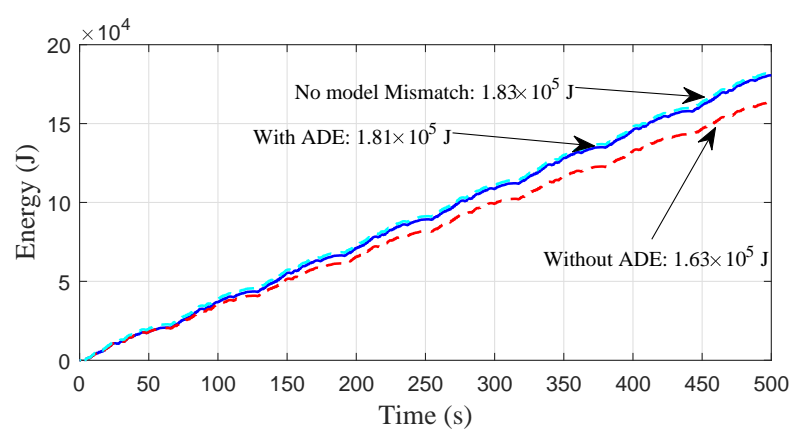

Fig. 13: Energy outputs: "With ADE" (Blue); "Without ADE" (Red); "No model mismatch" (Cyan).

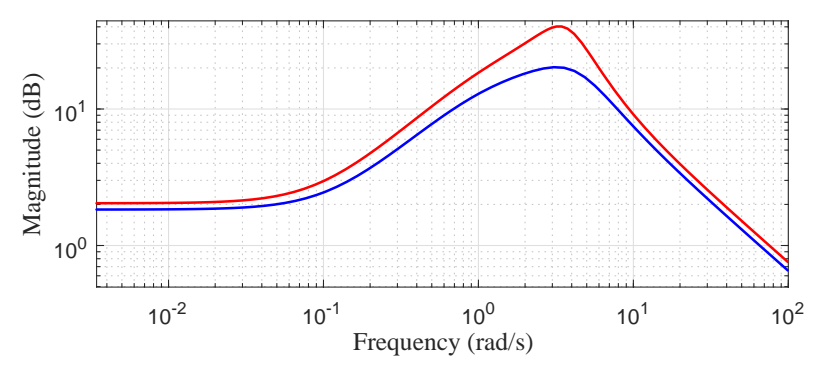

Fig. 14: Bode diagram of accurate $D_{r}(s)$ (39) (Red); inaccurate $\bar{D}_{r}(s)(42)$ (Blue).

duce the model mismatch for the radiation force dynamics. The following inaccurate model

$$
\bar{D}_{r}(s)=\frac{65.1 s^{2}+350 s+38.5}{1+6.5 s^{2}+24 s+21}
$$

is used for the controller design. The Bode diagrams of this model and the original model are compared in Fig. 14. To demonstrate the efficacy of the AHMPC, Fig. 15 shows the comparison of the energy outputs for 4 cases: when there are no model mismatch for both radiation force dynamics and excitation force dynamics, the maximum energy output is $1.83 \times 10^{5} \mathrm{~J}$; the mismatch of the radiation force dynamics can cause a slight decrease of energy output to $1.76 \times 10^{5} \mathrm{~J}$; when the excitation force has also model mismatch, the energy output drops to $1.46 \times 10^{5} \mathrm{~J}$. However, the proposed AHMPC framework can increase the energy output to $1.70 \times 10^{5} \mathrm{~J}$. This result shows: i) the proposed AHMPC can dramatically recover the control performance when the WEC system is subject to modeling uncertainties from both radiation force and excitation force, and ii) the radiation force estimation plays a less important role in influencing the energy output compared to the excitation force.

\section{CONCLUSiOnS}

A new adaptive hierarchical MPC framework consisting of a cascaded estimation of frequency-dependent dynamics for a WEC and a MPC for WEC control is proposed. To demonstrate the efficacy of the proposed framework, a simple and effective robust estimator is first developed to estimate the excitation force by assuming the radiation force dynamics is 


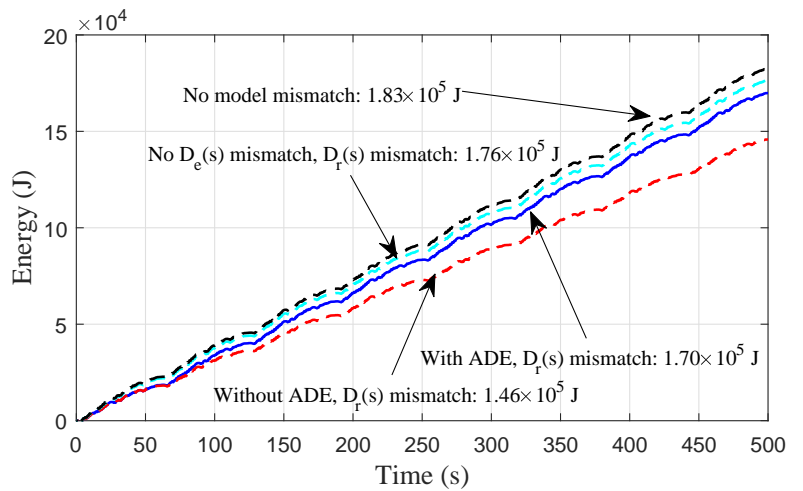

Fig. 15: Energy outputs: Accurate $D_{r}(s)$ and $D_{e}(s)$ (Black); Accurate $D_{e}(s)$ and inaccurate $D_{r}(s)$ (Cyan); inaccurate $D_{r}(s)$ and $D_{e}(s)$, but using an ADE mechanism to update $D_{e}(s)$ (Blue); inaccurate $D_{r}(s)$ and $D_{e}(s)$, no ADE mechanism (Red).

fixed, and then the estimated excitation force is used as the input to an adaptive law to estimate the unknown coefficients associated with the excitation force dynamics. This cascaded estimation method can achieve satisfactory estimation performance and can address the modeling uncertainties of the WEC dynamics due to the variations of sea conditions. Then the estimated WEC model is incorporated into a well-tailored MPC to maximize the energy output and guarantee the satisfaction of constraints. The proposed idea of estimating the excitation force can be used to deal with unknown radiation force in a similar way. Extensive simulations demonstrate and validate the efficacy of the proposed AHMPC framework. Although the point absorber is used as a case study, the proposed approach can be extended to the control of other types of WECs, and even other energy maximization control problems.

\section{REFERENCES}

[1] UK Parliament, "Renewable energy resources: Opportunities and constraints 1990-2020."

[2] I. Glendenning, "Ocean wave power," Applied Energy, vol. 3, no. 3, pp. $197-222,1977$.

[3] A. Clément and et al, "Wave energy in europe: current status and perspectives," Renewable and Sustainable Energy Reviews, vol. 6, no. 5, pp. $405-431,2002$.

[4] D. Bull and M. E. Ochs, "Technological cost-reduction pathways for attenuator wave energy converters in the marine hydrokinetic environment," Sandia National Laboratories, Tech. Rep., 2013, SAND20137207, Unlimited Release, Sandia Report.

[5] A. Babarit, G. Duclos, and A. H. Clément, "Comparison of latching control strategies for a heaving wave energy device in random sea," Applied Ocean Research, vol. 26, no. 5, pp. 227-238, 2004.

[6] F. d. O. António, "Phase control through load control of oscillating-body wave energy converters with hydraulic pto system," Ocean Engineering, vol. 35, no. 3-4, pp. 358-366, 2008.

[7] A. Babarit, M. Guglielmi, and A. H. Clément, "Declutching control of a wave energy converter," Ocean Engineering, vol. 36, no. 12-13, pp. $1015-1024,2009$.

[8] J. Falnes, Ocean waves and oscillating systems: linear interactions including wave-energy extraction. Cambridge university press, 2002.

[9] G. Li, G. Weiss, M. Mueller, S. Townley, and M. R. Belmont, "Wave energy converter control by wave prediction and dynamic programming," Renewable Energy, vol. 48, pp. 392-403, 2012.

[10] J. V. Ringwood, G. Bacelli, and F. Fusco, "Energy-maximizing control of wave-energy converters: The development of control system technology to optimize their operation," IEEE Control Systems, vol. 34, no. 5, pp. $30-55,2014$
[11] S. Zhan and G. Li, "Linear noncausal optimal control of wave energy converters," IEEE Transactions on Control System Technology, 2018.

[12] G. Li and M. R. Belmont, "Model predictive control of sea wave energy converters-part i: A convex approach for the case of a single device," Renewable Energy, vol. 69, pp. 453-463, 2014.

[13] S. Zhan, W. He, and G. Li, "Robust feedback model predictive control of sea wave energy converters," In proceedings of the 20th IFAC World Congress, Toulouse, France, 2017.

[14] G. Li, "Nonlinear model predictive control of a wave energy converter based on differential flatness parameterisation," International Journal of Control, vol. 90, no. 1, pp. 68-77, 2017.

[15] R. Genest and J. V. Ringwood, "A critical comparison of modelpredictive and pseudospectral control for wave energy devices," Journal of Ocean Engineering and Marine Energy, vol. 2, no. 4, pp. 485-499, 2016.

[16] N. Faedo, S. Olaya, and J. V. Ringwood, "Optimal control, mpc and mpc-like algorithms for wave energy systems: An overview," IFAC Journal of Systems and Control, vol. 1, pp. 37-56, 2017.

[17] F. Fusco and J. V. Ringwood, "A simple and effective real-time controller for wave energy converters," IEEE Transactions on Sustainable Energy, vol. 4, no. 1, pp. 21-30, 2013

[18] J. Na, G. Li, B. Wang, G. Herrmann, and S. Zhan, "Robust optimal control of wave energy converters based on adaptive dynamic programming," IEEE Transactions on Sustainable Energy, 2018.

[19] M. Richter, M. E. Magana, O. Sawodny, and T. K. A. Brekken, "Nonlinear model predictive control of a point absorber wave energy converter," IEEE Transactions on Sustainable Energy, vol. 4, no. 1, pp. 118-126, 2013.

[20] R. Genest, J. Davidson, and J. V. Ringwood, "Adaptive control of wave energy converters," IEEE Transactions on Sustainable Energy, 2018.

[21] Z. Liao, G. Nian, P. Stansby, and G. Li, "Control-oriented modelling for wave energy converter $\mathrm{m} 4$," in in proceedings of the 4th Asian Wave and Tidal Energy Conference, Taipei, Taiwan, 2018.

[22] F. Fusco and J. V. Ringwood, "Hierarchical robust control of oscillating wave energy converters with uncertain dynamics," IEEE Transactions on Sustainable Energy, vol. 5, no. 3, pp. 958-966, 2014.

[23] J. V. Ringwood, G. Bacelli, and F. Fusco, "Energy-maximizing control of wave-energy converters: The development of control system technology to optimize their operation," IEEE Control Systems Magazine, vol. 34, no. 5, pp. 30-55, 2014.

[24] M. Penalba, G. Giorgi, and J. V. Ringwood, "Mathematical modelling of wave energy converters: a review of nonlinear approaches," Renewable and Sustainable Energy Reviews, vol. 78, pp. 1188-1207, 2017.

[25] S.-R. Huang, H.-T. Chen, C.-H. Chung, C.-Y. Chu, G.-C. Li, and C.C. Wu, "Multivariable direct-drive linear generators for wave energy," Applied Energy, vol. 100, pp. 112 - 117, 2012.

[26] G. Weiss, G. Li, M. Mueller, S. Townley, , and M. R. Belmont, "Optimal control of wave energy converters using deterministic sea wave prediction," in The Energy \& Materials Research Conference, Torremolinos, Malaga, Spain, 2012.

[27] W. Cummins, "The impulse response function and ship motions," David Taylor Model Basin Washington DC, Tech. Rep., 1962.

[28] N. Faedo, Y. Peña-Sanchez, and J. V. Ringwood, "Finite-order hydrodynamic model determination for wave energy applications using momentmatching," Ocean Engineering, vol. 163, pp. 251-263, 2018.

[29] T. Pérez and T. I. Fossen, "Time-vs. frequency-domain identification of parametric radiation force models for marine structures at zero speed," Modeling, Identification and Control: A Norwegian Research Bulletin, vol. 29, no. 1, pp. 1-19, 2008.

[30] Z. Yu and J. Falnes, "State-space modelling of a vertical cylinder in heave," Applied Ocean Research, vol. 17, pp. 265-275, 1995.

[31] J. Na, A. S. Chen, G. Herrmann, R. Burke, and C. Brace, "Vehicle engine torque estimation via unknown input observer and adaptive parameter estimation," IEEE Transactions on Vehicular Technology, 2018.

[32] J. Na, X. Ren, and Y. Xia, "Adaptive parameter identification of linear siso systems with unknown time-delay," Systems \& Control Letters, vol. 66, pp. 43-50, 2014.

[33] Y. Pena-Sanchez, M. Garcia-Abril, F. Paparella, and J. V. Ringwood, "Estimation and forecasting of excitation force for arrays of wave energy devices," IEEE Transactions on Sustainable Energy, 2018.

[34] K. Hasselmann, T. Barnett, E. Bouws, H. Carlson, D. Cartwright, K. Enke, J. Ewing, H. Gienapp, D. Hasselmann, P. Kruseman et al., "Measurements of wind-wave growth and swell decay during the joint north sea wave project (jonswap)," Ergänzungsheft 8-12, 1973.

[35] E. Kristiansen, Å. Hjulstad, and O. Egeland, "State-space representation of radiation forces in time-domain vessel models," Ocean Engineering, vol. 32 , no. $17-18$, pp. 2195-2216, 2005. 


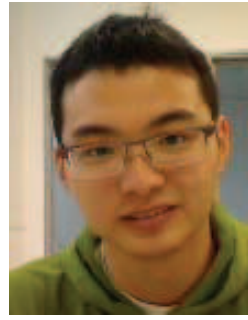

systems.

Siyuan Zhan (StM'18) received the bachelor's degree from Shanghai Jiao Tong University, Shanghai, China, in 2013, the master's degree from the University of Pennsylvania, Philadelphia, PA, USA, in 2014 and Ph.D. degree from the Queen Mary University of London, London, U.K., in 2018, respectively. He is currently a Postdoctoral Research Assistant with the Queen Mary University of London, London, U.K. His current research interests include constrained optimal control, adaptive control, model predictive control, and control applications in renewable energy

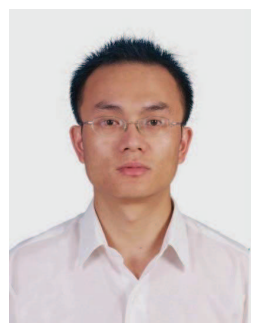

Jing Na (M'15) received the B.Sc. and Ph.D degrees from the School of Automation, Beijing Institute of Technology, Beijing, China, in 2004 and 2010, respectively. From 2011 to 2013, he was a Monaco/ITER Postdoctoral Fellow at the ITER Organization, Saint-Paul-lès-Durance, France. From 2015 to 2017, he was a Marie Curie Intra-European Fellow with the Department of Mechanical Engineering, University of Bristol, U.K. Since 2010, he has been with the Faculty of Mechanical and Electrical Engineering, Kunming University of Science and Technology, Kunming, China, where he became a Professor in 2013. He is currently an Associate Editor of Neurocomputing and has served as an international program committee Chair of ICMIC 2017. His current research interests include intelligent control, adaptive parameter estimation, nonlinear control and applications. Dr Na has been awarded the Best Application Paper Award of the 3rd IFAC International Conference on Intelligent Control and Automation Science (IFAC ICONS 2013), and the 2017 Hsue-shen Tsien Paper Award.

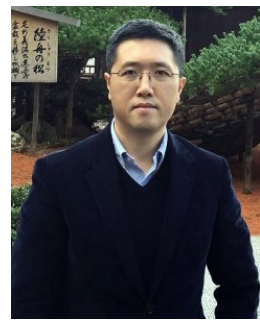

Guang Li (M'09) received his Ph.D. degree in Electrical and Electronics Engineering, specialized in control systems, from the University of Manchester, in 2007. He is currently a Lecturer in dynamics modelling and control in Queen Mary University of London, UK. His current research interests include constrained optimal control, model predictive control, adaptive robust control and control applications including renewable energies and energy storage, etc.

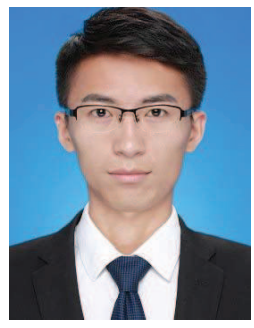

Bin Wang received the B.S. from Beijing Technology and Business University, Beijing, China, in 2016. He is currently pursuing the M.S. degree in Kunming University of Science and Technology, Kunming, China. His current research interests include adaptive dynamic programming, optimal control, parameter identification and prescribed performance control. 